\title{
Analysis of Application of the IOT Technology in the Power System in the 5G Era
}

\author{
Zang Yuwei, Xie Lianke, Ma Xingang, Gong Quanquan, Liu Hui
}

State Grid Shandong Electric Power Research Institute, Shandong Jinan, 250002

Keywords: 5G era; IOT technology; power system; application analysis

\begin{abstract}
As the 4G network has realized full coverage and the 5G era is within the reach, the Internet of Things will again achieve another qualitative leap and better help people improve their life quality and work efficiency. As an important part of social and economic development, the power system combined with the IOT technology in the 5G era can help to develop the power system stably and efficiently. However, how to integrate the IOT technology and the power system in the $5 \mathrm{G}$ era becomes an important subject. In view of this, the application of the IOT technology in the power system in the $5 \mathrm{G}$ era is analyzed in this paper based on the practice research.

With the rapid development of science and technology, mobile communication and network technology, the 5G era becomes possible and becomes a new industry fostered by the state. The advent of the 5G era means availability of ultra-high transmission rate, super large capacity bandwidth and low consumption network technology, and its integration with the IOT technology creates conditions for the development of the communication technology industry. Today, the Internet of things covers all over the life of all corners. Smart home, intelligent city and the like are all based on the IOT technology. And the IOT technology also promotes the power system to smart grid and gradually becomes an important technical support for the development of the smart grid.
\end{abstract}

\section{Integration with the IOT technology in the $5 \mathrm{G}$ era}

The IOT technology is to connect things with the Internet by means of wireless data communication, automatic identification and other technologies based on the computer network technology, so that things can be communicated independently via the network free from exterior environment influence, which has become a modern management model.

\subsection{IOT technology}

Comprehensive construction of the Internet of things with its technology development and innovation has become an important research subject in social construction and development. The Internet of things refers to the network connection between different objects. By 2D code, radio frequency, infrared induction and GPS based positioning

to identify the equipment, things are connected with the Internet in accordance with the protocol for information transmission and communication, to achieve network based positioning and management. In the IOT technology, the Internet is its root and extension. Based on the IOT technology, the core technology of IOT involves more complicated contents, such as GPS and RFID technology. The intelligent level of the power system has been improved obviously based on the IOT technology. With the application of network communication technology and high speed sensing technology, the power system is gradually transformed into the IOT power system.

\section{$1.25 G$ era}

The 4G era has basically satisfied people's network needs. However, the 5G era will realize further innovation on the basis of $4 \mathrm{G}$, to provide better services for people as the key point of mobile communication construction. The 5G era is characterized by the following points: first, ultra-high transmission efficiency and large capacity bandwidth. The IOT technology includes mobile terminals and intelligent devices. The mobile data traffic will run at more than thousand 
times the current speed. The 5G era will meet the traffic requirements. Second, billions of things are connected in the 4G ear, while the number will reach up to hundreds of billions in the 5G era. Third, low consumption and low latency: the IOT business carries on data transformation in line with the personalization principle, requiring the network to be highly applicable and specialized. Finally, the network based management is more intelligent and the frequency spectrum application rate increases. When the spectrum usage is increased, energy consumption will be reduced, thus saving cost with promotion of the IOT technology.

In addition, the advent of the IOT technology needs to meet the requirements as below: first, there should be sound network systems and communication protocols. Second, stability and security should be extremely high. The 5G era will provide conditions for connection of all things, so efficient and stable data transmission needs the Internet of things to have a strong sensor technology, identification technology and data processing capabilities. Only in this way can the IOT technology and the $5 \mathrm{G}$ era be combined to achieve a qualitative leap.

\section{Power system development}

With the improvement of people's living quality and the advancement of social productive forces, the demand for electric energy is increasing, which leads to the decrease of energy resources in China. Therefore, the smart grid has become the main way of power system construction and development. With the advent of information communication, UHV transmission technology and renewable resources, the power system is gradually moving towards the smart grid. The smart grid takes the UHV grid as the backbone, including power transmission and distribution, integrated with modern communication technology and power technology as an intelligentized and informationized solution, better meeting the requirement of connection between the power supply and the electrical equipment. The smart grid has a strong self-healing ability, which effectively improves the power system's stability and operational efficiency, as a new type of power system.

As for the development of the IOT technology, the smart grid is the basis of its development. The marriage of the IOT technology and the power system allows effective supervision of distribution line operations, residential electricity consumption and power grid running, helping technicians to acquire the real running state of the power system with real-time transmission of the data. In addition, the advent of the $5 \mathrm{G}$ era will further provide a resource for the development of the smart grid, to ensure the stable operation of the power system.

\section{Application of the IOT technology in the power system in the 5G era}

The smart grid is the core industry with massive application of IOT. According to the technology features of the $5 \mathrm{G}$ era and the development of the power grid, the technology of IOT has a wide development space in the power system. The application of the IOT technology in the 5G era can be based on power supply, substation, transmission and distribution, which is helpful for control over the power system operation.

\subsection{Electric power supply}

The advantages of the smart grid application focus on the use of clean energy, such as wind. According to the survey, the increasing grid output from wind power reached 6.01 million $\mathrm{kW}$ in 2017 in China and the grid-connected capacity reached up to 154 million $\mathrm{kW}$ as of the end of June. Even so, wind power is still faced with the problem of abandoned wind and power rationing, which is not conducive to economic improvement. At this stage, there are still some wind farms gaining no economic benefits. And the advent of the Internet of things allows installation of the sensor system in the fan equipment to get the factors influencing the fan output based on the sensor measurement technology and the data analysis technology, according to which in combination with the influence of the ambient temperature the wind output can be adjusted to prevent the problem of abandoned wind and power rationing. Based on the wireless communication technology, the dispatching center can know about the output state of the wind turbines with scientific scheduling to keep the power 
output in the limit and improve the utilization rate of clean energy.

\subsection{Power transformation}

With introduction of advanced intelligent equipment, the intelligent substation can collect data independently and carry out control, monitoring and measurement. However, actually the intelligent transformation is realized mainly by means of communication technology and the IOT technology. As for live-line overhaul, the equipment condition monitoring is connected effectively with the state evaluation via the Internet of things and 5G; the state data can be transmitted to the maintenance personnel during the maintenance process, effectively improving the overhaul efficiency. The underground cable fault prevention, judgment and treatment are all based on the IOT technology, which monitors the whole process of cable, collects the data and transports it to the monitoring center. And it also helps to scientifically evaluate the insulation state and the transmission state of the power grid. At present, some scholars have put forward the failure prevention technology, that is, comprehensive control is carried out over the power system by means of IOT object recognition, wireless communication and other technologies. It focuses on foreseeing the system failure and dealing with potential problems, to create conditions for the smooth operation of the power system.

\subsection{Power transmission}

In the transmission, the IOT technology based sensor is used to detect the transmission line and transmit the information about insulation conditions, line damages and other abnormal problems to the control center in real time, and helps the dispatcher to adjust the transmission system operation. Besides, the UAV is widely used for inspection of power transmission lines, based on the principle that the UAV is equipped with a camera, which helps inspectors know about the transmission line running conditions through wireless communication transmission by connecting the IOT technology to the UAV. In addition, an ultrasonic range-finder is installed on the UAV to realize the detection of transmission lines and help improve the accuracy of transmission line ranging. The installation of infrared sensors on the UAV can quickly locate the abnormal position of the transmission line. The advent of the 5G era enables the UAV to shoot and convey more clear images or videos, which will improve the stability of the inspection. With the technology of IOT, UAV based detection will be not restricted by environment or geographical location, which is helpful for transmission line monitoring.

\subsection{Power distribution process}

The distribution network is mainly connected with the residential electricity consumption, so there is a higher requirement for quality of the electric energy. With the popularization and application of the distributed generation (DG), the development of energy storage technology promotes the advancement of the automobile industry and the intelligent home system, and the active distribution network (ADN) is realized. The upgrading of DG access can realize power prediction and output adjustment by means of the IOT technology and improve the capacity of local consumption; besides, it can effectively connect the energy storage devices, DG, demand sides and other application layers, so that ADN deploys resources to reach the load and shift electricity from peak periods to off peak periods, thus lowering the economic input in the system. For example, the DG output tracking load curve is fluctuated to improve the system's power supply via the distributed generation in case of insufficient power supply. When the load is low, the power supply from energy storing devices can be used to ensure normal use. In addition, the technology of IOT can also help risk assessment, fault recovery and network reorganization to ensure the power restoration plan is scientific, and ADN can also improve the level of power supply and risk control by autonomous reorganization.

\subsection{Power utilization process}

The application of flexible load creates conditions for ADN management and control, which is combined with ADN movement management based on the IOT technology. For example, energy saving based on voltage lowering is supported by the IOT technology. In the power utilization 
identification of intelligent electric meters and advanced metering equipment, the voltage is lowered in real time to reduce the power consumption on the conditions of ensuring stable operation, which is helpful for achieving energy conservation and sustainable development. With the development of the automobile industry and increasing growth of automobile sales, connection with ADN and scientific management has become a key research subject for the future development. For example, the IOT technology makes it possible to analyze EV driving habits, load characteristics, control EV charge and discharge and generate a special operating mode according to the market power prices and ADN operating conditions.

The application of the IOT in the power system can optimize the transmission communication network and improve its stability and security, making it the core communication channel of the distribution network to make up for the functions that the modern public network and the wireless network hardly provide. And the Internet of things can control the power emergency conditions free from exterior environment influence, improving the risk-resistant communication system. The Internet of things also becomes the main way of information technology development in the future. At present, the Internet of things is not mature enough, and so is the IOT technology; for example, large research and economic investment, management and planning yet to be further upgraded are major factors hindering popularization of the Internet of things. Therefore, joint efforts from all parties are required to strengthen popularization and promotion of the Internet of things, thereby driving social and economic development.

\section{Conclusion}

In conclusion, with energy saving and sustainable strategic objectives put forward, the smart grid has been an important part of the power system development. With the requirements for the IOT power system met at present, the IOT technology has fully covered the nation. The development of the 5G era will further promote the development of the IOT technology; the combination of the IOT and $5 \mathrm{G}$ effectively connects the components of the power system to ensure a more stable and safer power system. The analysis in this paper is carried out in three areas, respectively: integration with the IOT technology in the 5G era, power system development and application of the IOT technology in the power system in the $5 \mathrm{G}$ era. I hope that it will be helpful for application of the IOT technology in the power system in the $5 \mathrm{G}$ era.

\section{References}

[1] Liu Chun. Research on Design and Implementation of Remote Meter Reading System Based on Internet of Things [J]. Computer Fans, 2017 (11).

[2] Wang Huafeng. On the Application of the IOT Technology in the Civil Air Defense System [J]. Information \& Communication, 2017 (10).

[3] Yang Jianfeng. Application of the IOT Technology in Fire Control Supervision and Management [J]. Electronic Technology \& Software Engineering, 2017 (17).

[4] Qin Jing. Research on Application of the IOT Technology in Elevators [J]. Construction Materials \& Decoration, 2017 (27).

[5] Zhao Shan, Jiang Lifen, Yi Yaping. Application and Prospect of the IOT Technology in Emergency Nursing [J]. Practical E-Journal of Clinical Nursing, 2017 (18).

[6] Zheng Xiaoqing. Analysis of Application of Cloud Computing in the Power Information Collection Based IOT Technology. [J]. China Computer \& Communication (Theory Section), 2017 (08).

[7] Wu Hua, Liu Honggang. Effective Application of the IOT Technology in Vehicle Management [J]. Technology Innovation and Application, 2017 (11).

[8] Li Qiang. Application of the IOT Technology in Energy Management Systems [J]. Technology 
Trend, 2017 (07).

[9] Hu Zhifeng. Research on Application of the IOT Technology in Camp Security [J]. China Computer \& Communication (Theory Section), 2017 (6). 